rheuma plus $2022 \cdot 21: 3$

https://doi.org/10.1007/s12688-022-00488-5 (c) The Author(s), under exclusive licence to Springer-Verlag GmbH Austria, ein Teil von Springer Nature 2022

\title{
Eine Ära geht zu Ende und es ist Zeit, Danke zu sagen!
}

Mit Ablauf des vergangenen Jahres endete die Funktionsperiode von Primarius Dr. Burkhard Leeb als Editor-in-Chief der Zeitschrift rheuma plus - und damit geht wahrlich eine Ära zu Ende. Hatte Prim. Leeb die Zeitschrift anfangs noch gemeinsam mit Doz. Attila Dunky herausgegeben, so übernahm er im Herbst 2011 als alleiniger Edior-in-Chief die Verantwortung dafür und prägte in den folgenden mehr als zehn Jahren ganz entscheidend die Ausrichtung von rheuma plus.

\section{) Geprägt war die Herausge- berschaft von Prim. Leeb durch fachliche Exzellenz und besonde- re Fallberichte}

Geprägt waren die Jahre der Herausgeberschaft von Prim. Leeb zum einen durch seine fachliche Exzellenz, zum anderen durch sein besonderes Interesse an Fallberichten: Trug ersteres ganz grundsätzlich dazu bei, dass die rheuma plus ihren Ruf als hochqualitatives Fortbildungsjournal kontinuierlich ausbauen konnte, so sicherte zweiteres - das Beisteuern von eigenen Fallberichten, aber auch das Motivieren von Mitarbeiter*innen sowie Kolleg*innen, solche $\mathrm{zu}$ verfassen - der Zeitschrift eine treue und begeisterte Leserschaft unter österreichischen Ärzt*innen.

Leser*innen der rheuma plus konnten sich aber nicht nur auf exzellente Fortbildungsartikel und anschauliche Fallberichte freuen, denn darüber hinaus wurden sie regelmäßig mit kontroversiellen Editorials eines Herausgebers versorgt, dem es nicht darum ging, sich selbst in Szene zu setzen sondern der zu eigenständigem und eigenwilligem Denken anregen und Diskussionen in Gang bringen wollte.
Abschließend und ausdrücklich zu betonen ist die Qualität der Zusammenarbeit mit dem Verlag: diese war stets unkompliziert sowie getragen von gegenseitigem Respekt und großer Wertschätzung - verbunden mit dem unerschütterlichen Vertrauen des Herausgebers, dass am Ende der nicht immer einfachen Produktionsprozesse ein ansehnliches Heft auf den Tischen der Empfänger liegen würde, zu dem diese auch gern griffen.

\section{》) Die Zusammenarbeit war stets getragen von gegenseitigem Respekt und großer Wertschät- zung}

Mir bleibt, mich im Namen des SpringerVerlags, aber auch ganz persönlich, sehr herzlich bei Prim. Dr. Burkhard Leeb für die ausgezeichnete Zusammenarbeit und sein großes und unermüdliches Engagement als Herausgeber zu bedanken und ihm alles Gute für die Zukunft zu wünschen. Es war eine große Ehre für uns, ihn zu unseren Herausgebern zählen zu dürfen.

Dr. Alois Sillaber

Geschäftsführer Springer-Verlag GmbH Wien

Hinweis des Verlags. Der Verlag bleibt in Hinblick auf geografische Zuordnungen und Gebietsbezeichnungen in veröffentlichten Karten und Institutsadressen neutral. 